\title{
Interfacial shear-stress effects on transient capillary wedge flow
}

\author{
Song-Kai Su and Chun-Liang Lai \\ Department of Mechanical Engineering, National Taiwan University, Taipei, Taiwan 106, Republic of China
}

(Received 13 October 2003; accepted 5 March 2004; published online 3 May 2004)

\begin{abstract}
The effects on the transient capillary flow in a wedge due to the interfacial shear-stress distribution $S$ along the flow direction $z$ is studied theoretically. With the assumptions of a slender liquid column and negligible gravitational and inertia effects, the problem is reduced to finding the axial velocity distribution at any cross section. The propagation of the liquid column $h(z, t)$ and the tip location $l(t)$ are then solved with the aid of the continuity equation. When the half-wedge angle $\alpha$, the contact angle $\theta$, and the shear-stress distribution on the free surface $S$ are constant, analytic solutions exist. Otherwise, numerical simulation has to be applied. The results indicate that when $S(z)$ is acting in the flow direction, the flow is strengthened and the liquid column propagates faster. When $S(z)$ is opposing the flow direction, reverse flow may exist near the free surface and the propagation speed of the liquid column is reduced. Moreover, for a capillary flow in a wedge with constant $\alpha$, $\theta$, and $S$, both the analytic solutions and the numerical simulation predict that $l(t) \propto t^{3 / 5}$ for the constant-flow-rate stage and $l(t) \propto t^{1 / 2}$ for the constant-height flow stage. When $S$ is a function of the flow direction $z$, the above functional relationship between $l$ and $t$ becomes no longer valid; it varies as the liquid column propagates along the wedge. (C) 2004 American Institute of Physics.
\end{abstract}

[DOI: $10.1063 / 1.1714791]$

\section{INTRODUCTION}

The capillary flows in a wedge arise in various industrial applications and are of technical interests. On earth, these flows are usually negligible or limited in extent due to the presence of gravity, as can be seen from the Bond number, $B o \equiv \Delta \rho \cdot g \cdot L^{2} / \sigma$, with $\Delta \rho$ denoting the density difference across the fluid interface; $g$, the gravity; $L$, the characteristic length scale, and $\sigma$, the surface tension. However, the capillary wedge flows still have various terrestrial applications such as flows in porous media and flows spreading along interior corners or grooves as faced in the oil industry, environmental technology, micro heat pipes, etc. In the microgravity environment of space, such flows and the capillary effect may become prevalent in fluid systems of large extent and possess particular importance in space technology. ${ }^{1,2}$ Inspace applications include all kinds of fluid management and control such as the handling and storage of fuel propellants and biological wastes, design of heat exchangers and heat pipes, etc.

Investigations on the capillary flows in interior corners, which are also called grooves or wedges, in the past four decades have covered problems of static interfacial shape, ${ }^{3-7}$ dynamic flow behavior, ${ }^{8-19}$ and instability phenomenon. ${ }^{20,21}$ The effects of the contact angle $(\theta)$ and wedge angle $(2 \alpha)$ on the capillary statics were well addressed in the literature. The spreading of the capillary flow along a wedge depends also on the contact angle and wedge angle. The criterion $\theta<\pi /$ $2-\alpha$ for a spontaneous wicking into wedges were derived by Concus and Finn, and is often referred to as the ConcusFinn condition. ${ }^{4,5}$ Since then, a great number of investigations have been devoted to the flow behavior and heat transfer in grooves both theoretically and experimentally.

The studies by Ayyaswamy, Catton, and Edwards ${ }^{8}$ and by Ransohoff and Radke ${ }^{15}$ both focused on the twodimensional hydrodynamic solution of a capillary flow in a wedge. In their analyses, different approaches to the dimensionless velocity distribution at any cross section along the flow direction were employed with the interfacial shear stresses neglected. The effects on the flow behavior due to the variation of the contact angle and wedge angle were discussed.

In the research by $\mathrm{Ma}$, Peterson, and $\mathrm{Lu},{ }^{12}$ an assumption of a constant velocity at the free surface was made and the velocity distribution at any cross section along the flow direction was obtained analytically. The friction factor was thus calculated numerically and the effects due to the vaporliquid interaction were discussed. Ma and Peterson then continued a series of analyses to further investigate the friction factor $^{13,14}$ and the heat transfer in a micro heat pipe. ${ }^{22,23}$

Catton and Stroes ${ }^{24}$ presented a semianalytical model for the heated inclined triangular capillary grooves. By the balance of the axial body force with the capillary effect, they concluded with a series of design curves, which can be applied to the estimation of the wetted length in the capillary grooves for a variety of operating conditions.

Most of the studies on the dynamics centered on the steady-state flow behavior and heat transfer. Weislogel and Lichter ${ }^{19}$ observed experimentally three stages in a transient capillary wedge flow: The start-up stage followed by a constant-flow-rate stage, then a constant-height flow stage. They also presented an asymptotic formulation on the limit of a slender liquid column with small inertia and solved analytically the tip location of a transient capillary flow in an interior corner for the latter two flow stages, i.e., the constant-flow-rate stage and the constant-height flow stage. Similarity solutions were also obtained by Romero and 


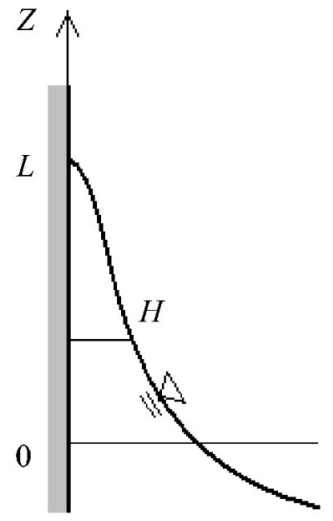

(a)

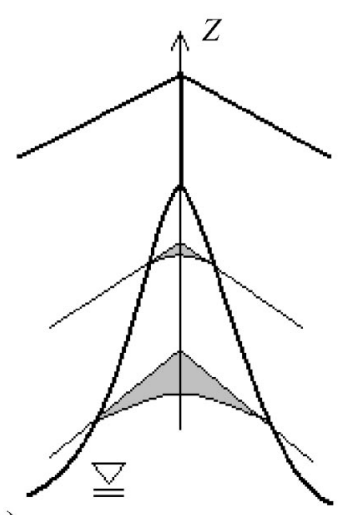

(b)

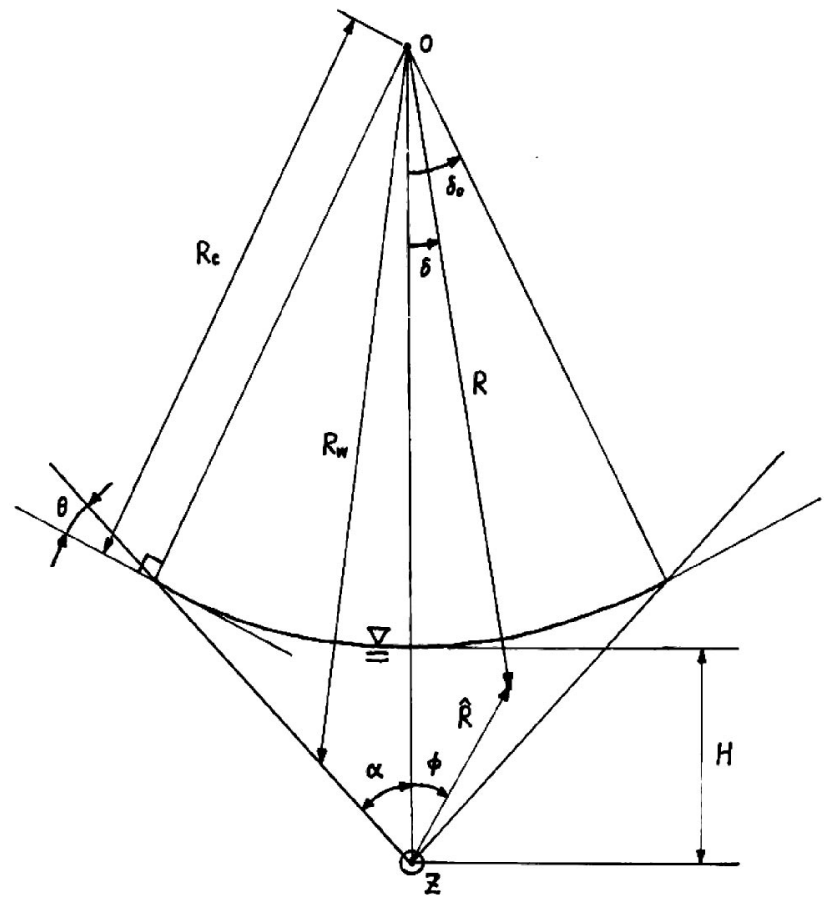

FIG. 2. The schematic diagram of a cross-section of the capillary wedge flow and the coordinate system employed.

(2) The gravitational effect is negligibly small.

(3) The liquid column is assumed to be slender. That is, the characteristic length scale in the transverse direction $H_{0}$ is small compared with that in the flow direction $L_{0}$.

(4) The lubrication approximation is applied to the wedge flow.

(5) A static contact angle is employed. This approximation is acceptable when the fluid velocity perpendicular to the contact line is small.

The second assumption can also be represented by a small value of Bond number, as introduced in last section. Assumption (4) implies that the mechanism of the capillary flow along a wedge is the balancing between the pressure gradient due to the capillary effect and the viscous force.

The slenderness of the flow configuration as indicated by assumption (3) possesses the following five implications:

(1) The surface curvature along the flow direction is negligibly small compared to that of any cross-section.

(2) The transverse flow velocities are small compared to the axial flow velocity $V_{Z}$ as can be derived from the scaling analysis of the continuity equation.

(3) The pressure distribution is uniform in the transverse direction across the liquid column. Thus, $P=P(Z, t)$ in the present study.

(4) The free surface at any cross section is a circular arc as a consequence of implication (3).

(5) Under the assumption of slenderness, the variation of $V_{Z}$ in the flow direction is much smaller than the variation in the transverse direction.

Consequently, the problem is reduced to the calculation of $V_{Z}$ at any cross section as a function of the wedge angle $\alpha$, the contact angle $\theta$, and the shear stress distribution $S$ at the 
free surface. The corresponding momentum equation is thus reduced to the following quasi-steady form:

$$
\frac{\partial^{2} V_{Z}}{\partial \hat{R}^{2}}+\frac{1}{\hat{R}} \frac{\partial V_{Z}}{\partial \hat{R}}+\frac{1}{\hat{R}^{2}} \frac{\partial^{2} V_{Z}}{\partial \phi^{2}}=\frac{1}{\mu}\left(\frac{d P}{d Z}\right) .
$$

The associated boundary conditions are as follows:

(1) No-slip boundary condition is applied at the solid wall.

(2) Since the geometry and, hence, the flow are symmetric with respect to the mid-plane of the wedge, the derivative of $V_{Z}$ in the azimuthal direction at the mid-plane must vanish.

(3) The shear stress distribution on the free surface needs to be satisfied.

Incorporated with the continuity equation, the propagation of the liquid column along the wedge, or the tip location $L$, can then be determined.

\section{B. Nondimensionalization and formulation}

In order to facilitate and generalize the discussion of the results of analysis, an appropriate nondimensionalization scheme needs to be employed. Let the lengths in the $\hat{R}$ - and $Z$-directions be scaled by $H_{0}$ and $L_{0}$, with $H_{0}$ representing a certain liquid height at the mid-plane of the wedge and $L_{0}$, a certain length of the liquid column in the Z-directions. With the assumption of slenderness, we then have $\epsilon \equiv H_{0} / L_{0} \ll 1$. The cross-sectional area is scaled by $H_{0}^{2}$. The pressure is scaled by $\left(\sigma / R_{0}\right)$ with $\sigma$ denoting the surface tension and $R_{0}$, the characteristic radius of the free surface. Time is scaled by $\left(L_{0} / W_{0}\right)$ with $W_{0}$ denoting the characteristic velocity in the Z-direction, which is determined, according to Eq. (1), from the balance between the viscous effect and the pressure gradient in the flow direction, i.e., $W_{0}$ $=P_{0} H_{0}^{2} / \mu L_{0}$. However, as suggested by Weislogel and Lichter, ${ }^{19} W_{0}$ is modified as $P_{0} H_{0}^{2} \sin ^{2} \alpha / \mu L_{0}$ to account for the geometric effect. The present study will thus adopt this expression for $W_{0}$.

In order to convert the expression for the reference pressure in terms of $H_{0}$, certain geometric relations need to be derived first. For the convenience of mathematical manipulation and subsequent numerical calculations, a polar coordinate system located at the center of the circular arc of the free surface will be employed. The azimuthal angle and the radial position are denoted, respectively, by $\delta$ and $R$ in this coordinate system, as shown in Fig. 2. Let $R_{c}$ denote the radius of the free surface and $R_{w}$, the radial distance from the new coordinate center to the wedge walls. It can be shown that

$$
R_{w}(\delta)=R_{c} \cdot \frac{\cos \theta}{\sin (\alpha+\delta)} .
$$

Accordingly, the liquid height at the centerline of any cross section $H$ can be related to $R_{c}$ as

$$
H=R_{w}(\delta=0)-R_{c}=R_{c} \cdot\left(\frac{\cos \theta}{\sin \alpha}-1\right)=R_{c} \cdot f^{-1},
$$

with

$$
f=\left(\frac{\cos \theta}{\sin \alpha}-1\right)^{-1},
$$

a geometry factor. Therefore,

$$
R_{c}=f \cdot H
$$

The reference pressure $P_{0}$ can now be expressed in terms of $H_{0}$ as

$$
P_{0}=\sigma / f H_{0},
$$

with $R_{0}=f \cdot H_{0}$.

Consequently, we have $W_{0}=\sigma \cdot \epsilon \sin ^{2} \alpha / \mu \cdot f$.

To further predict the propagation of the liquid column or the dimensionless tip location of the liquid column, the following dimensionless form of the mass conservation is employed:

$$
\frac{\partial A}{\partial t}=-\frac{\partial}{\partial z}\left(A \cdot \bar{v}_{Z}\right),
$$

where $A$ is the cross-sectional area and $\bar{v}_{Z}$, the averaged $v_{Z}$-velocity. By defining

$$
A=2 \cdot \int_{0}^{\delta_{0}} \int_{r_{c}}^{r_{w}} r d r d \delta=F_{A} \cdot h^{2},
$$

with

$$
F_{A} \equiv f^{2} \cdot\left(\frac{\sin 2 \delta_{0}}{2}+\frac{\sin ^{2} \delta_{0}}{\tan \alpha}-\delta_{0}\right),
$$

Eq. (7) can be rewritten as follows:

$$
\frac{\partial}{\partial t}\left(F_{A} \cdot h^{2}\right)=-\frac{\partial}{\partial z}\left(F_{A} h^{2} \cdot \bar{v}_{z}\right) .
$$

In the above expressions, $r_{c}=R_{c} / H_{0}=f h, r_{w}=R_{w} / H_{0}=r_{c}$ $\cdot \cos \theta / \sin (\alpha+\delta)$, and $\delta_{0}=\pi / 2-\alpha-\theta$, the azimuthal angle of the contact line.

Furthermore, since $\bar{v}_{Z}$ can be expressed in terms of $h_{z}$ as

$$
\bar{v}_{Z}=-F_{i} \cdot h_{Z},
$$

which will be derived in Sec. III A with $F_{i}$ defined as the flow coefficient, Eq. (10) is reduced to

$$
\frac{\partial}{\partial t}\left(F_{A} \cdot h^{2}\right)=\frac{\partial}{\partial z}\left(F_{A} \cdot F_{i} \cdot h^{2} h_{z}\right)
$$

For flow situations with constant wedge angle and contact angle, $F_{A}$ is a constant and thus Eq. (12) can be further reduced to

$$
2 \frac{\partial h}{\partial t}=2 F_{i}\left(\frac{\partial h}{\partial z}\right)^{2}+\frac{\partial F_{i}}{\partial z} \cdot h \frac{\partial h}{\partial z}+F_{i} \cdot h \frac{\partial^{2} h}{\partial z^{2}} .
$$

Equation (13) is then used to calculate the propagation of the liquid column and predict the tip location, $l$. In order to do so, $F_{i}$ and thus the velocity distribution at any cross section $V_{Z}$ need to be determined first and are discussed in next section. The details of the numerical computation of the tip locations will be presented in Sec. IV. 


\section{VELOCITY DISTRIBUTION AND FLOW COEFFICIENT}

From the momentum equation and the associated boundary conditions as listed in Sec. II, the velocity distribution at any cross section of a capillary wedge flow will be solved numerically. For the convenience of numerical computations, the polar coordinate system located at the center of the circular arc of the free surface is used. The azimuthal angle and the radial coordinate are denoted respectively by $\delta$ and $R$, as shown in Fig. 2. The momentum equation, i.e., Eq. (1), is then rewritten as, in this coordinate system,

$$
\frac{\partial^{2} V_{Z}}{\partial R^{2}}+\frac{1}{R} \frac{\partial V_{Z}}{\partial R}+\frac{1}{R^{2}} \frac{\partial^{2} V_{Z}}{\partial \delta^{2}}=\frac{1}{\mu}\left(\frac{d P}{d Z}\right) .
$$

With the nondimensionalization scheme introduced in Sec. II B, Eq. (14) is further reduced to

$$
\frac{\partial^{2} v_{Z}}{\partial r^{2}}+\frac{1}{r} \frac{\partial v_{Z}}{\partial r}+\frac{1}{r^{2}} \frac{\partial^{2} v_{Z}}{\partial \delta^{2}}=\frac{1}{\sin ^{2} \alpha} \frac{d p}{d z} .
$$

To further simplify Eq. (15), the following transformation is applied:

$$
\begin{aligned}
& \xi=\frac{r}{r_{c}}, \\
& w=v_{Z} \cdot \frac{\sin ^{2} \alpha}{r_{c}^{2} \cdot\left(-\frac{d p}{d z}\right)} .
\end{aligned}
$$

The z-component momentum equation at any cross section of the wedge flow then becomes as follows:

$$
\frac{\partial^{2} w}{\partial \xi^{2}}+\frac{1}{\xi} \frac{\partial w}{\partial \xi}+\frac{1}{\xi^{2}} \frac{\partial^{2} w}{\partial \delta^{2}}+1=0 .
$$

The associated boundary conditions are

$$
\begin{aligned}
& \frac{\partial w}{\partial \xi}=S(z) \quad \text { at } \xi=\frac{r_{c}}{r_{c}}=1, \\
& w=0 \quad \text { at } \quad \xi=\frac{r_{w}}{r_{c}}=\frac{\cos \theta}{\sin (\alpha+\delta)}, \\
& \frac{\partial w}{\partial \delta}=0 \quad \text { at } \delta=0 .
\end{aligned}
$$

Equation (19) indicates a shear stress distribution, represented by the dimensionless parameter $S(z)$, along the free surface in the flow direction. Equations (20) and (21) denote, respectively, the no-slip condition at the solid walls and the symmetry condition at the mid-plane of the wedge.

As described in Sec. II B, in order to simulate the propagation of the liquid column in a wedge, the average velocity $\bar{v}_{Z}$ has to be represented in terms of the centerline height $h(z, t)$ as indicated by Eq. (11). From Eq. (17), $\bar{v}_{Z}$ can be expressed as follows:

$$
\bar{v}_{Z}=\bar{w} \cdot \frac{r_{c}^{2} \cdot\left(-\frac{d p}{d z}\right)}{\sin ^{2} \alpha} .
$$

Since the free surface at any cross section is a circular arc with a radius $R_{c}$, the pressure gradient $d p / d z$ is reduced to, with the aid of Eq. (5),

$$
\frac{d p}{d z}=\frac{d}{d z}\left(p_{\infty}-\frac{\sigma / R_{c}}{P_{0}}\right)=-\frac{d}{d z}\left(\frac{1}{h}\right)=\frac{1}{h^{2}} \frac{d h}{d z} .
$$

The expression of $\bar{v}_{Z}$ then becomes, with the substitution of Eq. (23) into Eq. (22),

$$
\bar{v}_{Z}=-\left(\frac{\bar{w} \cdot f^{2}}{\sin ^{2} \alpha}\right) \cdot \frac{d h}{d z},
$$

or

$$
\bar{v}_{Z}=-F_{i} \cdot h_{Z},
$$

with

$$
F_{i}=\frac{\bar{w} \cdot f^{2}}{\sin ^{2} \alpha} .
$$

Since the average velocity $\bar{v}_{Z}$ is proportional to $F_{i}$, it is therefore named $F_{i}$ the flow coefficient in the present study.

Equation (11) indicates that $\bar{v}_{Z}$ is determined solely by the slope of the centerline height $h_{Z}$. As a result, there may exist a finite propagation speed even at the tip of the liquid column where $h=0$ and $h_{Z}$ possesses a finite value.

Equation (18) is then solved numerically followed by the calculations of the average velocity $\bar{w}$ and the flow coefficient $F_{i}$ for various combinations of the half-wedge angle $\alpha$, the contact angle $\theta$, and the shear-stress parameter $S$. Equation (13) is hence employed to calculate the propagation of the liquid column along the wedge.

To solve $w(\xi, \delta)$, Eq. (18) was first cast into a centralspaced finite difference equation and calculated numerically with the associated boundary conditions, i.e., Eqs. (19)-(21). Supplementary numerical computations were then performed on various grid systems, which suggested that satisfactory grid independence could be achieved with a $(40 \times 40)$-grid. The numerical results were further justified through comparison of $F_{i}$ with those obtained by Weislogel and Lichter. ${ }^{19}$

The flow situation with $S=0.0$ will be used to study the effects of $\alpha$ and $\theta$ on the flow motion. The computed results of $\bar{w}$ and $F_{i}$ are listed in Table I with the variation of $F_{i}$ also shown in Fig. 3. Note that the Concus-Finn condition has to be always satisfied by any assignment of $\alpha$ and $\theta$. It can be learned from Fig. 3 that although $F_{i}$ always increases with $\theta$ for any fixed value of $\alpha$, it reaches a minimum value first and then ascends as $\alpha$ increases for a fixed value of $\theta$. The results are in good consistency with those obtained by Weislogel and Lichter, ${ }^{19}$ which thus justifies the validity of the present analysis and numerical calculations.

The dimensionless parameter $S$ represents the interfacial shear stress at each cross section of a capillary wedge flow. According to the condition shown in Eq. (19), a negative value of $S$ indicates the interfacial shear stress is acting in the flow direction and, consequently, the flow is strengthened. With a positive value of $S$, the interfacial shear stress is opposing the flow motion and a reverse flow may exist near the free surface if $S$ is large enough. 
TABLE I. The computed results of $\bar{w}$ and $F_{i}$ as functions of $\alpha$ and $\theta$ for flow situation with $S=0.0$.

\begin{tabular}{|c|c|c|c|c|c|c|}
\hline \multirow[b]{2}{*}{$\alpha$} & \multicolumn{2}{|c|}{$\theta=0^{\circ}$} & \multicolumn{2}{|c|}{$\theta=10^{\circ}$} & \multicolumn{2}{|c|}{$\theta=20^{\circ}$} \\
\hline & $\bar{w}$ & $F_{i}$ & $\bar{w}$ & $F_{i}$ & $\bar{w}$ & $F_{i}$ \\
\hline 5 & $1.32 \mathrm{E}-01$ & 0.1584 & $1.28 \mathrm{E}-01$ & 0.1588 & $1.16 \mathrm{E}-01$ & 0.1593 \\
\hline 10 & $1.04 \mathrm{E}-01$ & 0.152 & $1.00 \mathrm{E}-01$ & 0.1524 & $8.98 \mathrm{E}-02$ & 0.1531 \\
\hline 20 & $6.09 \mathrm{E}-02$ & 0.1407 & $5.84 \mathrm{E}-02$ & 0.1413 & $5.10 \mathrm{E}-02$ & 0.1427 \\
\hline 30 & $3.03 \mathrm{E}-02$ & 0.1329 & $3.15 \mathrm{E}-02$ & 0.134 & $2.63 \mathrm{E}-02$ & 0.136 \\
\hline 40 & $1.64 \mathrm{E}-02$ & 0.1285 & $1.52 \mathrm{E}-02$ & 0.1302 & $1.17 \mathrm{E}-02$ & 0.1331 \\
\hline 45 & $1.09 \mathrm{E}-02$ & 0.1275 & $9.99 \mathrm{E}-03$ & 0.1297 & $7.20 \mathrm{E}-03$ & 0.1331 \\
\hline 50 & $6.96 \mathrm{E}-03$ & 0.1272 & $6.22 \mathrm{E}-03$ & 0.13 & $4.05 \mathrm{E}-03$ & 0.1342 \\
\hline 60 & $2.31 \mathrm{E}-03$ & 0.1288 & $1.88 \mathrm{E}-03$ & 0.1336 & $7.58 \mathrm{E}-04$ & 0.1397 \\
\hline 65 & $1.15 \mathrm{E}-03$ & 0.1306 & $8.45 \mathrm{E}-04$ & 0.1371 & $1.61 \mathrm{E}-04$ & 0.1446 \\
\hline 70 & $4.84 \mathrm{E}-04$ & 0.133 & $2.89 \mathrm{E}-04$ & 0.1422 & & \\
\hline 75 & $1.58 \mathrm{E}-04$ & 0.1358 & $5.33 \mathrm{E}-05$ & 0.1494 & & \\
\hline 80 & $3.20 \mathrm{E}-05$ & 0.1388 & & & & \\
\hline \multirow[t]{2}{*}{85} & $2.05 \mathrm{E}-06$ & 0.1415 & & & \multirow{2}{*}{\multicolumn{2}{|c|}{$\theta=50^{\circ}$}} \\
\hline & \multicolumn{2}{|c|}{$\theta=30^{\circ}$} & \multicolumn{2}{|c|}{$\theta=40^{\circ}$} & & \\
\hline$\alpha$ & $\bar{w}$ & $F_{i}$ & $\bar{w}$ & $F_{i}$ & $\bar{w}$ & $F_{i}$ \\
\hline 5 & $9.70 \mathrm{E}-02$ & 0.1598 & $7.40 \mathrm{E}-02$ & 0.1605 & $4.98 \mathrm{E}-02$ & 0.1612 \\
\hline 10 & $7.38 \mathrm{E}-02$ & 0.154 & $5.44 \mathrm{E}-02$ & 0.155 & $3.44 \mathrm{E}-02$ & 0.1561 \\
\hline 15 & $5.48 \mathrm{E}-02$ & 0.1487 & $3.86 \mathrm{E}-02$ & 0.15 & $2.23 \mathrm{E}-02$ & 0.1514 \\
\hline 20 & $3.96 \mathrm{E}-02$ & 0.1442 & $2.62 \mathrm{E}-02$ & 0.1458 & $1.33 \mathrm{E}-02$ & 0.1475 \\
\hline 25 & $2.77 \mathrm{E}-02$ & 0.1407 & $1.68 \mathrm{E}-02$ & 0.1425 & $7.00 \mathrm{E}-03$ & 0.1444 \\
\hline 30 & $1.85 \mathrm{E}-02$ & 0.1381 & $9.92 \mathrm{E}-03$ & 0.1402 & $2.90 \mathrm{E}-03$ & 0.1423 \\
\hline 35 & $1.17 \mathrm{E}-02$ & 0.1365 & $5.14 \mathrm{E}-03$ & 0.1388 & $6.76 \mathrm{E}-04$ & 0.1411 \\
\hline 40 & $6.77 \mathrm{E}-03$ & 0.1359 & $2.10 \mathrm{E}-03$ & 0.1385 & & \\
\hline 45 & $3.44 \mathrm{E}-03$ & 0.1363 & $4.83 \mathrm{E}-04$ & 0.1391 & & \\
\hline 50 & $1.38 \mathrm{E}-03$ & 0.1378 & & & & \\
\hline \multirow[t]{2}{*}{55} & $3.09 \mathrm{E}-04$ & 0.1405 & & & \multirow{2}{*}{\multicolumn{2}{|c|}{$\theta=80^{\circ}$}} \\
\hline & \multicolumn{2}{|c|}{$\theta=60^{\circ}$} & \multicolumn{2}{|c|}{$\theta=70^{\circ}$} & & \\
\hline$\alpha$ & $\bar{w}$ & $F_{i}$ & $\bar{w}$ & $F_{i}$ & $\bar{w}$ & $F_{i}$ \\
\hline 5 & $2.76 \mathrm{E}-02$ & 0.162 & $1.06 \mathrm{E}-02$ & 0.1629 & $1.23 \mathrm{E}-03$ & 0.1641 \\
\hline 10 & $1.68 \mathrm{E}-02$ & 0.1573 & $4.50 \mathrm{E}-03$ & 0.1588 & & \\
\hline 15 & $8.90 \mathrm{E}-03$ & 0.153 & $1.07 \mathrm{E}-03$ & 0.1548 & & \\
\hline 20 & $3.73 E-03$ & 0.1493 & & & & \\
\hline 25 & $8.77 \mathrm{E}-04$ & 0.1464 & & & & \\
\hline
\end{tabular}

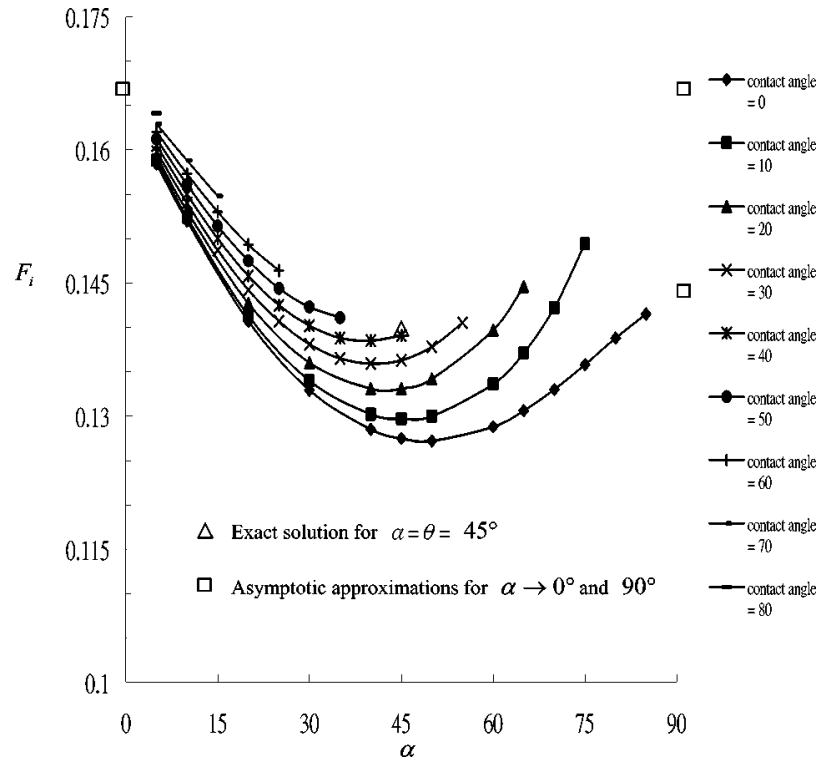

FIG. 3. Variation of $F_{i}$ with $\alpha$ and $\theta ; S=0.0$. Exact solution for $\alpha=\theta=45^{\circ}$ and asymptotic approximations for $\alpha$ approaching $0^{\circ}$ and $90^{\circ}$ [Weislogel and Lichter (Ref. 19)] are also included for comparison as references.
Shown in Fig. 4 are the flow distributions for $S=0.05$, 0.075 , and 0.1 with $\alpha=45^{\circ}$ and $\theta=30^{\circ}$. It can be seen that when $S=0.05$, the reverse flow starts to occur from the upper corner near the free surface where the velocity and momentum of the liquid originally driven by the capillary effect are smallest due to the large viscous resistance therein resulted from the no-slip boundary condition at the adjacent solid wall. As $S$ increases, the reverse flow region extends toward the central portion of the cross section of the wedge flow and becomes larger and larger.

Consequently, the average velocity $\bar{w}$ and the flow coefficient $F_{i}$ at a cross section will change as $S$ varies. Presented in Fig. 5 are the variations of $\bar{w}$ and $F_{i}$ with $S$ for $\alpha=45^{\circ}$ and $\theta=30^{\circ}$. It can be seen that both $\bar{w}$ and $F_{i}$ decrease linearly with $S$. It is also indicated in the figure that when $S \approx 0.1$, the region of the reverse flow becomes so large that both $\bar{w}$ and $F_{i}$ turn to be negative values. Of course, in practical situations $S$ is usually a small value.

To conclude, the variations of $\alpha, \theta$, and $S$ will result in a variation of $F_{i}$ and, thus, affect the flow motion and the 


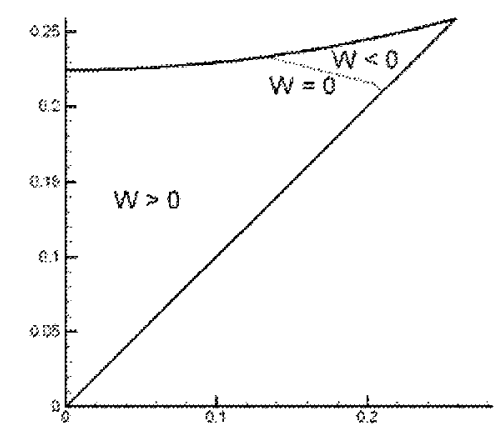

(a)

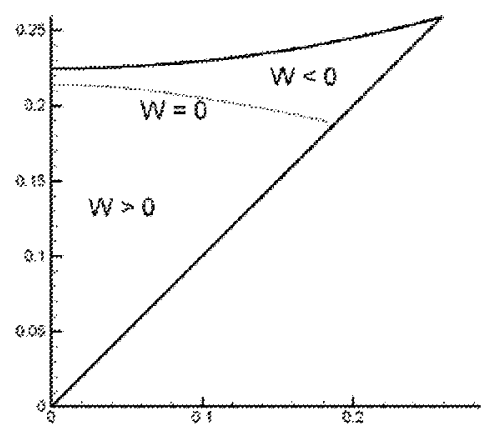

(b)

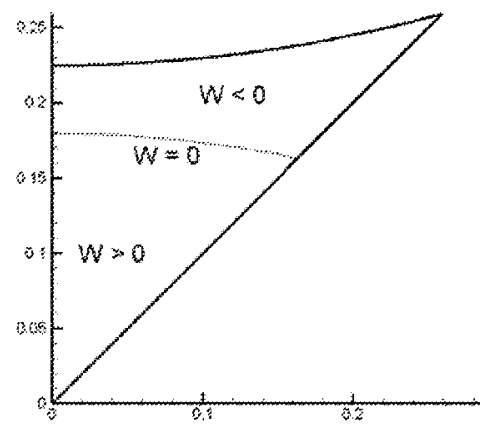

(c)

FIG. 4. The $w$-distributions for $\alpha=45^{\circ}, \theta=30^{\circ}$ : (a) $S=0.05$; (b) $S=0.075$; (c) $S=0.1$.

propagation of the liquid column along a wedge. The analysis will be given in next section.

\section{PROPAGATION AND TIP LOCATIONS OF THE LIQUID COLUMN}

\section{A. Numerical simulation}

The shear stresses acting on the gas-liquid interface and the variation of the wedge angle have great influence on the motion of capillary flow in a wedge, and are crucial to the controlling of the wedge flow or the heat transfer in a micro heat pipe. The present work is focused on the interfacial shear-stress effect on the capillary flow in a wedge with a constant wedge angle. The shear stresses imposed on the free surface of a wedge flow might be considered, as an example, as the result due to the viscous interaction between the liquid and vapor in a micro-tube. The gaseous phase, flowing in the
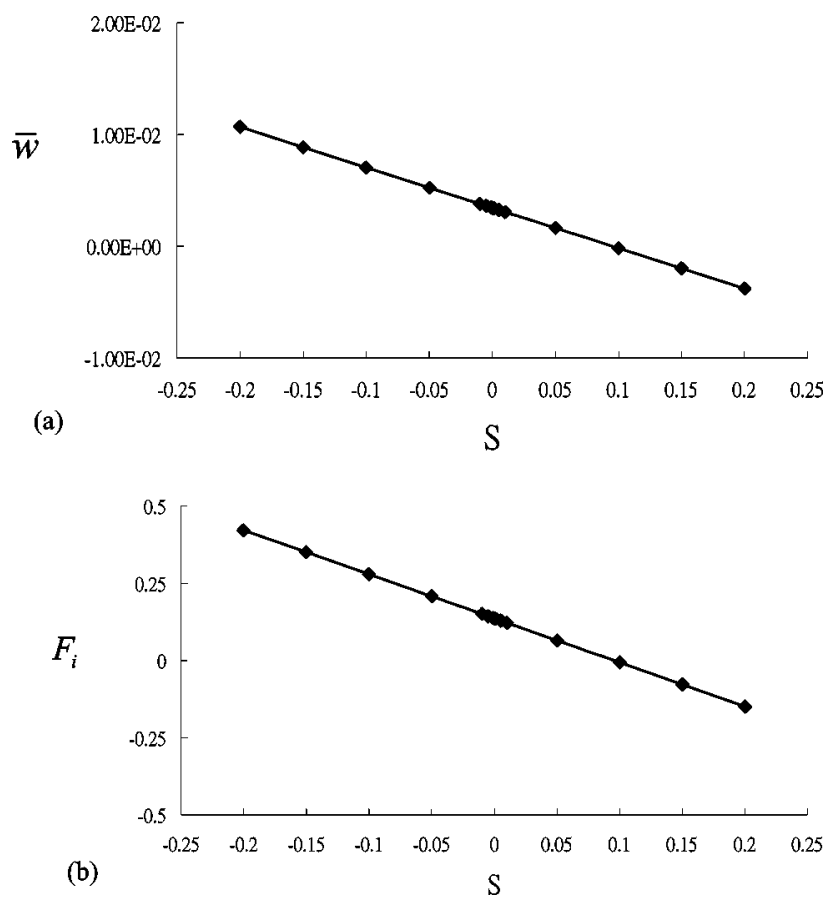

FIG. 5. The variations of $\bar{w}$ and $F_{i}$ with $S$ for $\alpha=45^{\circ}$ and $\theta=30^{\circ}$ : (a) $\bar{w}$ vs $S$; (b) $F_{i}$ vs $S$. same or opposite direction of the liquid flow, will result in, respectively, an acceleration or a deceleration of the wedge flow.

With $\alpha$ and $\theta$ both assumed constant, the shape factor $F_{A}$ is independent of $z$ and hence Eq. (13) will be applied in the subsequent discussion. By assuming the interfacial shear stresses be constant at each cross section but vary with $z$, the shear-stress parameter $S$ is then a function of $z$, i.e., $S$ $=S(z)$. Since the flow coefficient $F_{i}=F_{i}(\alpha, \theta, S)$, it is also a function of $z$. Accordingly, the similarity solutions suggested by Weislogel and Lichter ${ }^{19}$ become inapplicable. For convenience, Eq. (13) is repeated in the following:

$$
2 \frac{\partial h}{\partial t}=2 F_{i}\left(\frac{\partial h}{\partial z}\right)^{2}+\frac{\partial F_{i}}{\partial z} \cdot h \frac{\partial h}{\partial z}+F_{i} \cdot h \frac{\partial^{2} h}{\partial z^{2}} .
$$

To solve Eq. (13), the flow coefficient function $F_{i}(z)$ must be specified beforehand. As discussed in Sec. III and shown in Fig. 5, $F_{i}$ varies linearly with $S$ for fixed $\alpha$ and $\theta$. The following linear approximation is, therefore, assumed:

$$
F_{i}(z)=c_{1}+c_{2} S(z),
$$

with $c_{1}$ and $c_{2}$ being two constants to be determined from the curve fitting of the $\left(F_{i}, S\right)$ data, i.e., Fig. 5(b). Actually, the interfacial shear-stress distribution along the flow direction, i.e., $S(z)$, has to be solved from the coupled equations of the gaseous phase and the liquid column. However, in order to understand the effect on the transient capillary flow motion in a wedge due to the variation of interfacial shear stresses, a linear distribution of $S(z)$ is assumed simply for the purpose of demonstration, i.e.,

$$
S(z)=c_{3}+c_{4} \cdot z \text {. }
$$

In the above equation, $c_{3}$ and $c_{4}$ are arbitrary constants to be specified in accord with the physical situations to be demonstrated. For example, for $S(z)>0$ with $c_{3}>0$ and $c_{4}<0$, Eq. (27) indicates an decreasing interfacial shear-stress distribution acting in the $(-z)$-direction due to a counter gaseous flow opposing the liquid motion. If $S(z)<0$ with $c_{3}=0$ and $c_{4}<0$, Eq. (27) then represents a gaseous flow in the same direction of the liquid motion with an increasing interfacial shear-stress distribution. With the flow coefficient function determined from Eqs. (26) and (27), Eq. (13) is a nonlinear 
partial differential equation of $h(z, t)$ which is to be solved numerically to simulate the propagation of the liquid column in a wedge.

Several important aspects regarding the numerical simulation of Eq. (13) need to be addressed first. They are (1) linearization of the nonlinear terms $(\partial h / \partial z)^{2}, h(\partial h / \partial z)$, and $h\left(\partial^{2} h / \partial z^{2}\right)$; (2) specification of the initial condition, i.e., the initial distribution of $h(z)$; (3) establishment of the grid system; (4) specification of the boundary conditions for the constant-flow-rate stage and the constant-height stage, respectively; (5) determination of the tip location of the liquid column $l$; (6) re-scope of the computational domain to accommodate the propagation of the liquid column.

The strategies and methods to deal with the above aspects are described detailedly in the following:

(1) Linearization of the nonlinear terms:

Before the finite difference formulation is carried out, Eq. (13) needs to be linearized first as follows:

$$
\begin{aligned}
& \left(\frac{\partial h}{\partial z}\right)^{2} \approx\left(\frac{\partial h}{\partial z}\right)_{n-1}\left(\frac{\partial h}{\partial z}\right)_{n}, \\
& h\left(\frac{\partial h}{\partial z}\right) \approx \frac{1}{2} h_{n-1}\left(\frac{\partial h}{\partial z}\right)_{n}+\frac{1}{2} h_{n}\left(\frac{\partial h}{\partial z}\right)_{n-1}, \\
& h \frac{\partial^{2} h}{\partial z^{2}} \approx \frac{1}{2} h_{n-1}\left(\frac{\partial^{2} h}{\partial z^{2}}\right)_{n}+\frac{1}{2} h_{n}\left(\frac{\partial^{2} h}{\partial z^{2}}\right)_{n-1} .
\end{aligned}
$$

In the above expressions, the subscripts $(n-1)$ and $n$ denote, respectively, the values at the previous and present time steps.

(2) Specification of the initial distribution:

An input of the initial distribution of $h(z)$ is needed to solve Eq. (13). Since the present analysis does not study the flow motion from the start-up stage, any specification of the initial distribution of $h(z)$ will be an approximation. In the present study, the initial condition is given from the results of the similarity solutions for the constant-flow-rate stage or the constant-height stage, respectively. The numerical computation is then employed to study the interfacial shear-stress effects on the subsequent propagation of the liquid column.

(3) Establishment of the grid system:

Before establishing the whole grid system in $(z, t)$-plane, certain definitions need to be introduced first. Let $t(j)$ denote the present time instant; $l(t(j))$, the length of liquid column at time $t(j) ; m(j)$, the number of space interval totally covered by the liquid column at time $t(j)$. We then have

$$
t(j)=t(0)+j \cdot \Delta t, \quad j=0,1,2, \ldots, n
$$

and

$$
\begin{aligned}
& z(i)=0+i \cdot \Delta z, \\
& i=0,1,2, \ldots, m(j)-1, m(j), m(j)+1, \ldots, k-1, k,
\end{aligned}
$$

with

$$
\Delta z=(l(t(0))-0) / m(0) .
$$

In Eq. (32), the parameter $k$ is a given integer which determines the permissible computational domain in space, i.e.,
$[0, z(k)]$, and covers at least three times of the initial liquidcolumn length. Moreover, from the definition of $m(j)$,

$$
h(z(i), t(j))=0 \quad \text { for } i \geqslant m(j+1), \quad j=0,1,2, \ldots, n
$$

and

$$
m(j) \geqslant m(j-1),
$$

because of the propagation of the liquid column as time proceeds. In general, the tip of the liquid column is not located exactly at the grid point except at the initial stage when $z(m(0))=l(t(0))$ and $h(z(m(0), t(0))=0$ by assignment. Once the grid system is determined, the linearized form of Eq. (13) can then be cast into a finite difference equation with a forward-time, central-spaced scheme.

(4) Specification of the boundary conditions:

Since Eq. (13) is a second-order partial differential equation in variable $z$, two boundary conditions are needed to solve the equation. However, because the tip location of the liquid column is not exactly at the grid point as it propagates, the boundary condition of $h(z(m(j)), t(j))=0$ becomes inappropriate for the corresponding finite difference equation and cannot be applied in the numerical computation. Instead, $h(z(m(j)), t(j))$ will be approximated by a linear extrapolation from $h(z(m(j)-2), t(j))$ and $h(z(m(j)-1), t(j))$, which then provides an extra equation for computation to deal with one of the boundary conditions. Another boundary condition to be satisfied depends on the flow situation, i.e., the constant-flow-rate stage or the constant-height stage.

(a) The constant-flow-rate stage:

Since the constant-flow-rate condition implies that $(d / d t) \int_{0}^{l(t)} h^{2}(z, t) d z$ is a constant, the corresponding finite difference equation of this condition is as follows:

$$
\begin{array}{r}
\frac{1}{\Delta t}\left[\sum_{i=0}^{m(j)} h^{2}(z(i), t(j))-\sum_{i=0}^{m(j-1)} h^{2}(z(i), t(j-1))\right]=C, \\
j=0,1,2, \ldots n,
\end{array}
$$

in which $C$ is a constant to be calculated from the similarity solution. Equation (36) then provides another equation for computation.

(b) The constant-height stage:

The other boundary condition of Eq. (13) for the constant-height condition is simply described by

$$
h(z(0), t(j))=h(z(0), t(0)), \quad j=0,1,2, \ldots, n,
$$

where $h(z(0), t(0))$ is the initial height of the liquid column at $z(0)$ attained from the similarity solution.

(5) Determination of the tip location, $l$ :

Since the tip location of the liquid column is not located exactly at the grid point, as discussed previously, it will be approximated by a linear extrapolation from $h(z(m)(j)$ $-1), t(j))$ and $h(z(m(j)), t(j))$. That is, the tip location, $l$, will be determined by the intercept of the straight line with a slope $h(z(m(j)), t(j))-h(z(m(j)-1), t(j)) / z(m(j))$ $-z(m(j)-1)$ and the $z$-axis.

(6) Re-scope of the computational domain:

The permissible computational domain of space is $[0, z(k)]$ while the real computational domain of space is $[0, z(m(j))]$ which is the maximum length totally covered by 


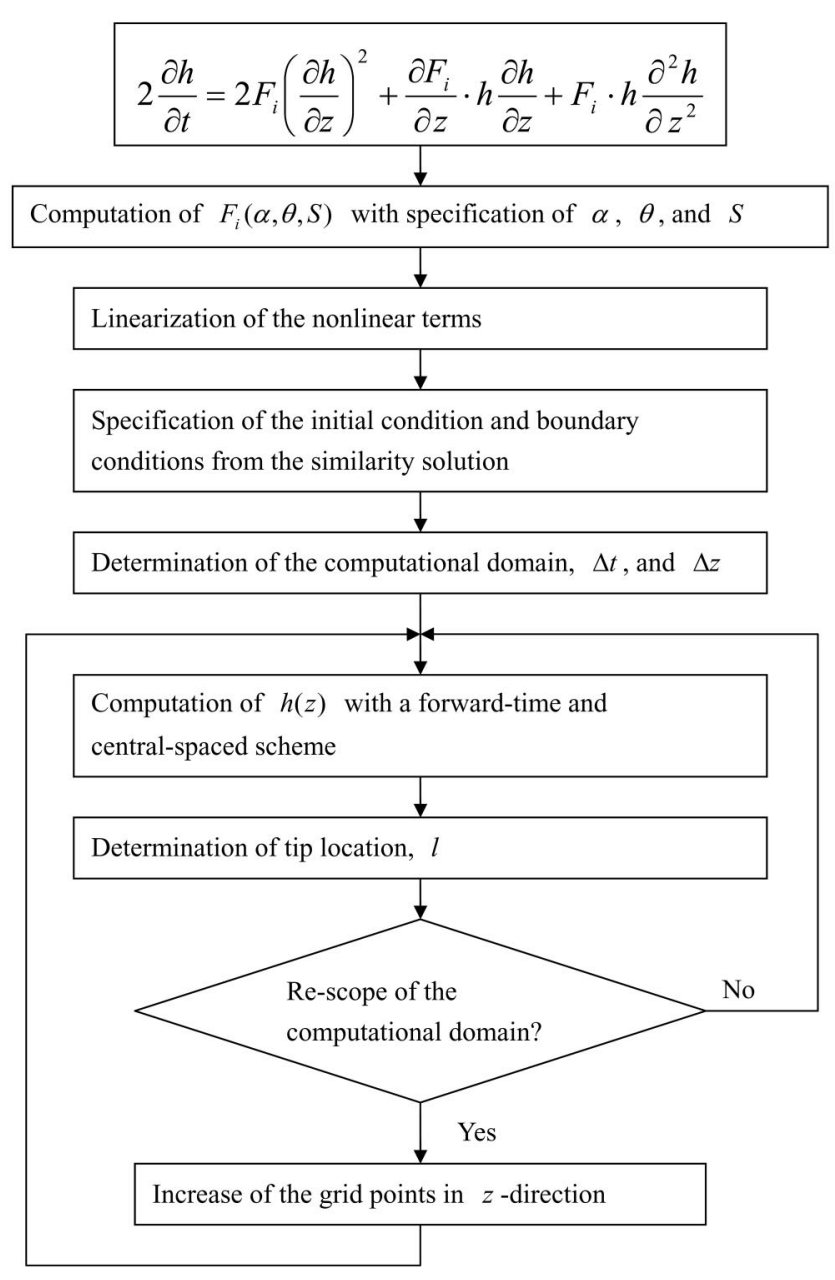

FIG. 6. Flow chart for the numerical calculations of $h(z, t)$ and $l(t)$.

the liquid column, as defined in strategy (3). Since the liquid column keeps increasing as time proceeds, the re-scope of the real computational domain becomes necessary. A criterion based on the tip location is applied to determine the necessity of the re-scope. If the tip location determined by the linear extrapolation as presented in strategy (5) is over one grid space $\Delta z$, the computational domain will be increased accordingly; otherwise, it will remain the same. We, thus, have $m(j) \geqslant m(j-1)$.

A flow chart illustrating the numerical procedure incorporating the above strategies and methods to solve Eq. (13) is included in Fig. 6. With the numerical scheme described above, the distribution of $h(z, t)$ and the propagation of $l(t)$ are then computed for the constant-flow-rate and constantheight stages.

\section{B. Results and discussion of the flow motion}

In this section, the variations of $h(z, t)$ and $l(t)$ for different values of the half-wedge angle $\alpha$, the contact angle $\theta$, and a linear shear distribution of $S(z)$ are investigated by numerical simulation. The results are then presented and discussed systematically.

In order to attain an accurate and stable numerical solution of $h(z, t)$ with reasonable computational time, an appro-
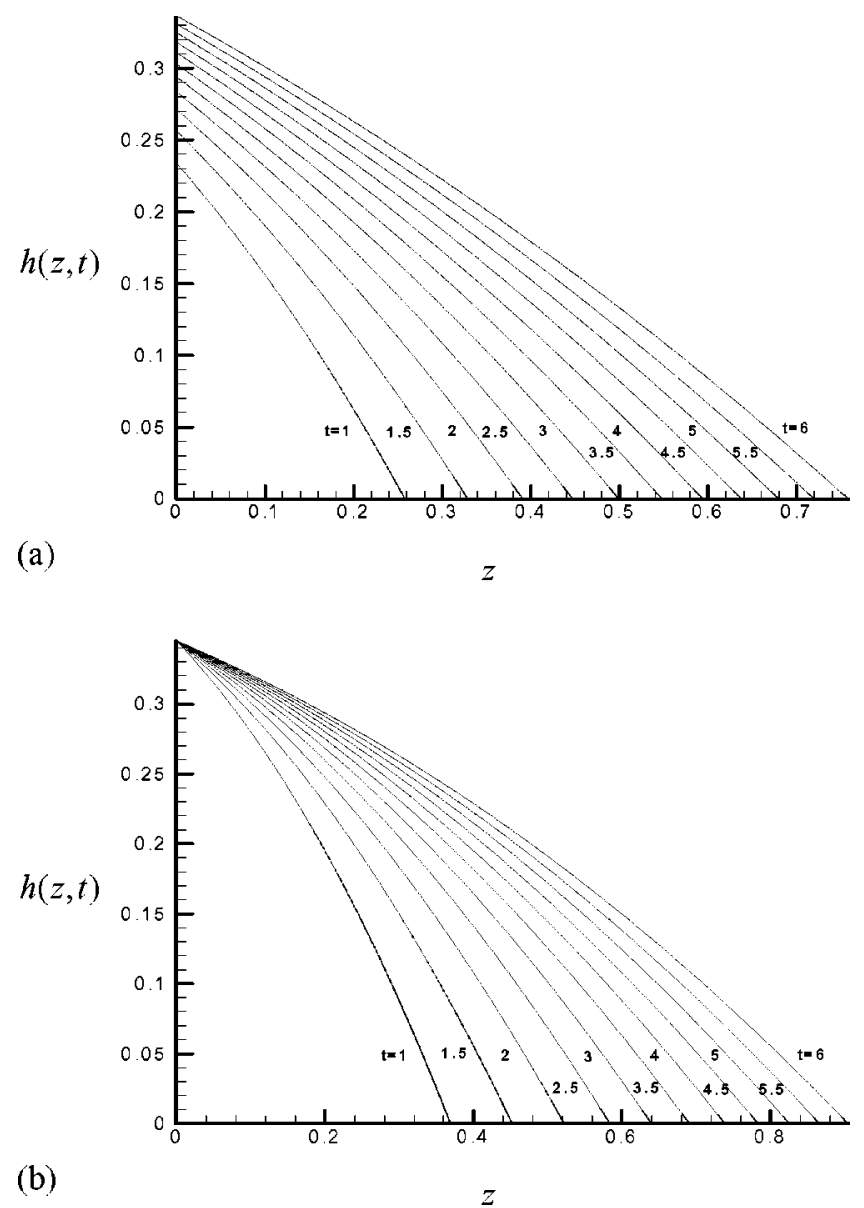

FIG. 7. The variations of $h(z, t)$ from the numerical simulation with $\alpha=45^{\circ}$, $\theta=30^{\circ}$, and $S=0.0$ : (a) The constant-flow-rate stage; (b) the constant-height flow stage.

priate grid system is needed. In the following, a capillary wedge flow with $\alpha=45^{\circ}, \theta=30^{\circ}$, and $S=0.0$ will be used for the grid independence test.

As discussed in the previous section, the space interval $\Delta z$ is determined by the specification of the value of $m(0)$, i.e., $\Delta z=l(t(0)) / m(0)$. Thus, computations with $m(0)$ $=50,100,200,300,400$, and 500 and with $\Delta t=0.1$ are performed for comparison. It is concluded that satisfactory results can be achieved with $m(0)=400$ for the constantflow-rate stage and $m(0)=300$ for the constant-height flow stage.

With the space interval determined above, computations with $\Delta t=0.05,0.1,0.5$, and 1.0 are performed to examine the appropriateness of the time interval. The tip locations $l(t)$ computed at certain time instants are compared with those predicted by the similarity solution. A reasonable time interval with $\Delta t=0.1$ is thus selected for the subsequent computations.

\section{A free shear-stress distribution, $S=0.0$}

In this subsection, the propagation and tip location of the liquid column along a wedge with $\alpha=45^{\circ}, \theta=30^{\circ}$, and $S$ $=0.0$ are simulated by the numerical method developed in last section. The distributions of $h(z, t)$ at different time instants are shown in Fig. 7; Fig. 7(a) is for the constant-flow- 

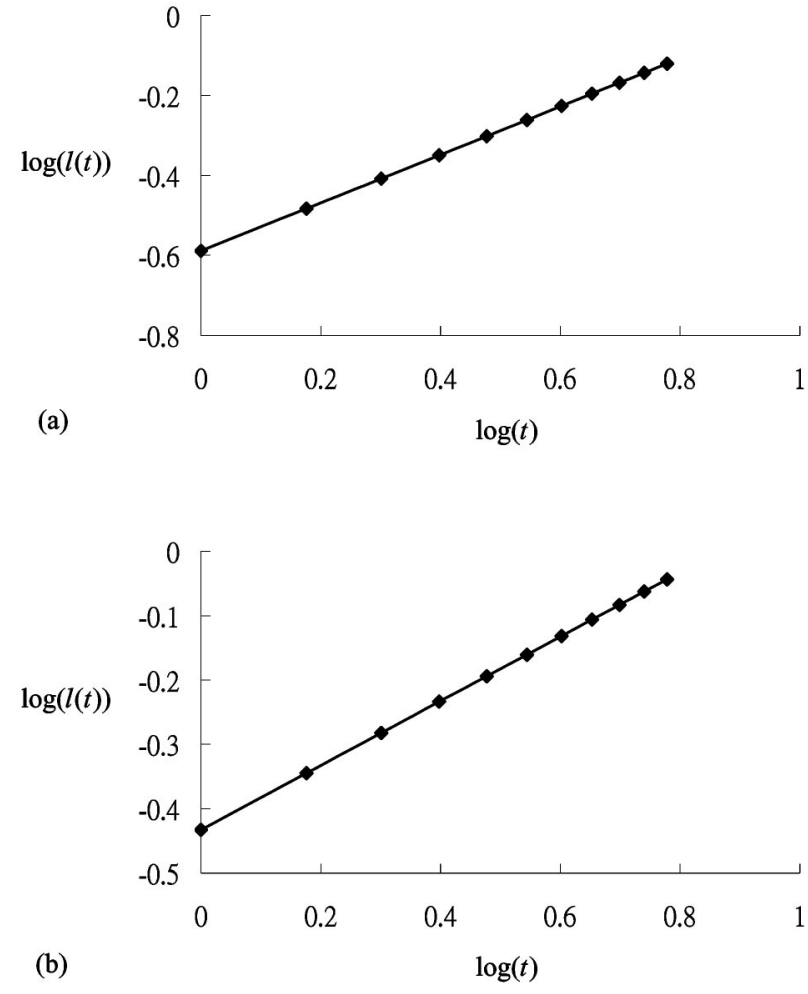

FIG. 8. The relationships between $l(t)$ and $t$ with $\alpha=45^{\circ}, \theta=30^{\circ}$, and $S$ $=0.0$ : (a) the constant-flow-rate stage; (b) the constant-height flow stage.

rate stage and Fig. 7(b) is for the constant-height flow stage. The corresponding tip locations as a function of time are plotted in Figs. 8(a) and 8(b), respectively. In Fig. 7(a), the liquid heights at $z=0$, i.e., $h(0, t)$, approach to each other as time proceeds, which indicates that the flow possesses a tendency to move from the constant-flow-rate stage toward the constant-height flow stage, as observed experimentally. ${ }^{19}$ The $\log -\log$ diagram in Fig. 8 also indicates a functional relationship between the tip location $l$ and time $t$, i.e., $l(t)$ $\propto t^{3 / 5}$ and $l(t) \propto t^{1 / 2}$ for the constant-flow rate stage and the constant-height flow stage, respectively. These results are identical to those predicted by the similarity solutions. The validity and accuracy of the numerical scheme developed in the present study is thus justified.

\section{A constant shear-stress distribution, $S=$ constant}

A capillary wedge flow with a constant shear-stress distribution imposed on the free surface is studied in this subsection. From the definition of $S$, a negative value of $S$ represents a shear-stress distribution acting in the flow direction and a positive value of $S$ is in the direction opposing the wedge flow. Since $\alpha, \theta$, and $S$ are independent of the flow direction $z$, the flow coefficient $F_{i}(\alpha, \theta, S)$ is a constant. Thus, similarity solutions exist. The numerical method developed in last section is also applicable.

The variation of $h(z, t)$ and the corresponding tip locations as a function of time for different values of $\alpha, \beta$, and $S$ are all similar to those shown in Figs. 7 and 8. The results indicate that: (1) For whatever combination of $\alpha, \theta$, and $S$, a large value of the flow coefficient $F_{i}$ correspond to a larger propagation speed of the liquid column. (2) When the shearstress distribution on the free surface is acting in the flow direction, the capillary wedge flow is strengthened while when the shear stresses are opposing the flow, reverse flows may occur near the free surface, Fig. 4, and the capillary wedge flow is retarded. As a result, with a negative value of $S$, the flow coefficient $F_{i}$ and thus the propagation speed of the liquid column becomes larger while with a positive value of $S$, they are smaller compared to those obtained with a free shear condition. (3) Both the similarity solution and the numerical simulation predict $l(t) \propto t^{3 / 5}$ for the constant-flowrate stage and $l(t) \propto t^{1 / 2}$ for the constant-height flow stage. This fact justifies once again the validity of the numerical method developed in this study.

\section{A linear shear-stress distribution, $S=c_{3}+c_{4} \cdot z$}

The shear-stress distribution imposed on the free surface of a capillary wedge flow is to simulate the interaction between the liquid flow and the adjacent vapor flow. If the vapor is flowing in the same or opposite direction of the capillary wedge flow, a negative or positive value of $S(z)$ is specified correspondingly. In general, the shear-stress distribution is a function of the flow direction $z$. However, since the correct shear-stress distribution must be attained from solving the coupled equations of the liquid and vapor flow, any specification of the shear-stress distribution on the freesurface will be an approximation. Therefore, a linear distribution of $S(z)$ will be assumed in the following analysis simply for the purpose of demonstration of the essential physics. In addition, as discussed in Section 3, there exists a linear relation between $F_{i}$ and $S$, which, derived from the curve fitting of the data shown in Fig. 5, gives

$$
F_{i}=0.1363-1.4277 \cdot S(z) \text {. }
$$

Since $F_{i}(z)$ is a function of $z$, similarity solutions, in general, may not exist and the numerical simulation of Eq. (13) has to be employed.

The following four distributions of $S(z)$ will be used in the subsequent computations and analysis:

$$
\begin{aligned}
& S(z)=0.0+0.1 \cdot z, \\
& S(z)=0.075-0.1 \cdot z, \\
& S(z)=-0.1+0.1 \cdot z, \\
& S(z)=0.0-0.1 \cdot z .
\end{aligned}
$$

Equations (39) and (40) represent, respectively, an increasing and decreasing shear-stress distribution acting in the $-z$-direction to simulate a counter vapor flow. Equations (41) and (42) denote, respectively, a decreasing and increasing shear-stress distribution acting in the $+z$-direction to simulate a vapor flow in the same direction of the capillary wedge flow.

With the $S$-distribution described by Eq. (39), the variations of $h(z, t)$ and the tip location $l(t)$ are similar to those with a free shear, i.e., Figs. 7 and 8 . However, the functional relationship between the tip location and time is no longer 


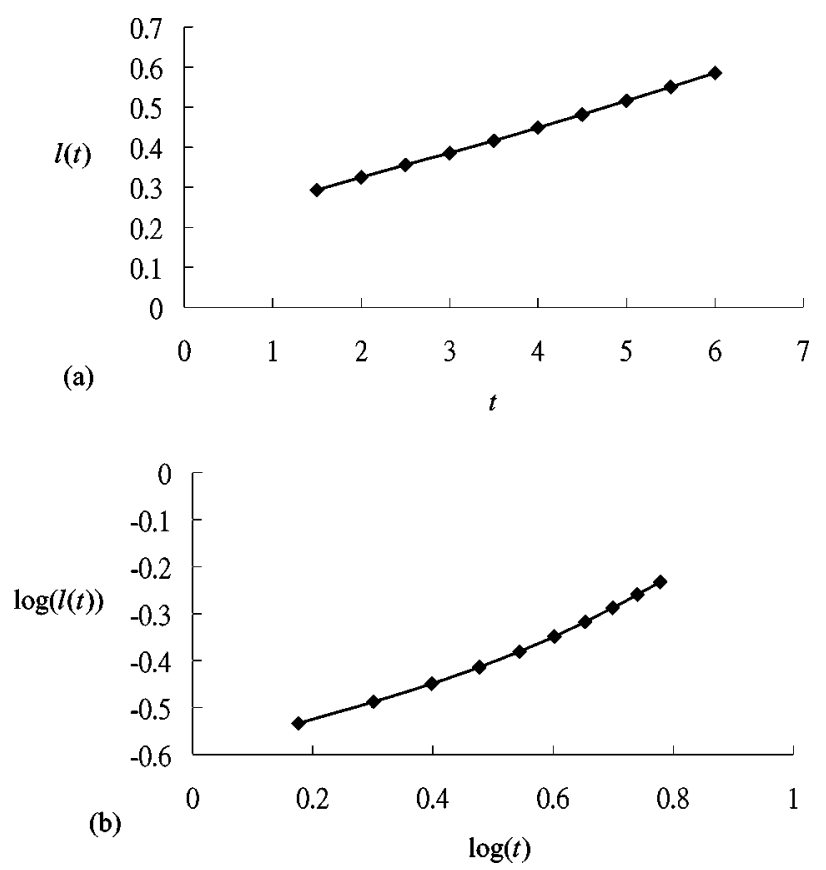

FIG. 9. The relation between $l(t)$ ant $t$ for the constant-flow-rate stage with $\alpha=45^{\circ}, \theta=30^{\circ}$, and $S(z)=0.075-0.1 \cdot z$.

$l(t) \propto t^{3 / 5}$ for the constant-flow-rate stage and no longer $l(t)$ $\propto t^{1 / 2}$ for the constant-height flow stage; it varies as the liquid column propagates, e.g., Fig. 9. In average,

$$
l(t) \propto t^{0.500},
$$

for the constant-flow-rate stage, and

$$
l(t) \propto t^{0.374},
$$

for the constant-height flow stage.

The variations of $h(z, t)$ and $l(t)$ with the $S$-distribution described by Eq. (40) are then computed for the two flow stages. Due to the variation of $S(z)$, the functional relationship between $l$ and $t$ varies along the flow direction. In average,

$$
l(t) \propto t^{0.502},
$$

for the constant-flow-rate stage, and

$$
l(t) \propto t^{0.420},
$$

for the constant-height flow stage.

It can be learned from the above results that: (1) For the $S$ - distributions as described by Eqs. (39) and (40) with $S$ $>0$, the functional relationship between $l$ and $t$ becomes milder, i.e., the power of $t$ is smaller than that with a constant $S$. This result indicates that, with an opposing interfacial shear-stress distribution, the propagation speed of the liquid column becomes slower. (2) When the opposing shear stresses become weaker as the liquid column propagates downstream, the distribution of $h(z, t)$ tends to change from a convex to a concave shape, as shown in Fig. 10.

Results computed with Eqs. (41) and (42) are obtained in a similar way. Once again, the functional relationship between $l$ and $t$ varies as the liquid column propagates due to

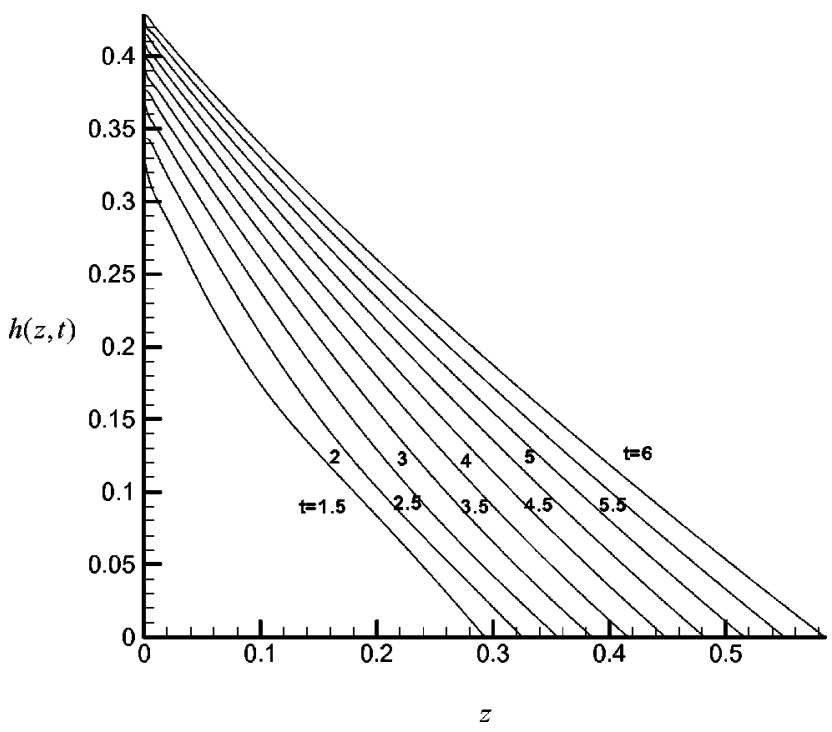

FIG. 10. The variation of $h(z, t)$ for the constant-flow-rate stage with $\alpha=45^{\circ}, \theta=30^{\circ}$, and $S(z)=0.075-0.1 \cdot z$.

the variation of $S(z)$. In the average, when computed with Eq. (41), a decreasing shear-stress distribution acting in the $+z$-direction,

$$
l(t) \propto t^{0.611},
$$

for the constant-flow-rate stage, and

$$
l(t) \propto t^{0.534},
$$

for the constant-height flow stage. When computed with Eq. (42), an increasing shear-stress distribution acting in the $+z$-direction,

$$
l(t) \propto t^{0.677,}
$$

for the constant-flow-rate stage, and

$$
l(t) \propto t^{0.587},
$$

for the constant-height flow stage.

The results indicate that: (1) For the $S$-distribution as described by Eqs. (41) and (42) with $S<0$, the functional relationship between $l$ and $t$ becomes stronger, i.e., the power of $t$ is larger than that with a constant $S$. (2) When the shear stresses acting in the flow direction become stronger as the liquid column propagates downstream, the distribution of $h(z, t)$ tends to change from a convex to a concave shape, similar to Fig. 10 but milder.

Although the propagation of the liquid column and tip location are calculated and predicted for a transient capillary wedge flow, there are certain limitations of the model applied. Because of the assumption of slenderness, the model cannot be applied to the start-up stage. Only the constantflow-rate stage and the constant-height stage, which were suggested from experimental observations, are analyzed. As a result of the above limitations, there is a lack in the initial conditions for the prediction of the liquid column propagation and has to resort to the similarity solutions as an approximation. In addition, the linear relationship between the flow coefficient $F_{i}$ and the interfacial shear stress distribution $S(z)$ as indicated by Eq. (38) is only valid for small values of 
$S(z)$ with $\alpha=45^{\circ}$ and $\theta=30^{\circ}$. The coefficient in the equation have to be changed for different values of $\alpha$ and $\theta$. The numerical scheme needs then to be modified accordingly. A more complete model which can predict the liquid column propagation of a capillary wedge flow from the start-up stage becomes therefore very realistic and important. Moreover, in order to investigate more precisely the interaction between the liquid column propagation and the adjacent gaseous flow, a model and numerical scheme need to be developed so that the coupling between these two phases can be simulated appropriately.

\section{CONCLUSIONS}

The effects on the transient capillary flow in a wedge due to the interfacial shear-stress distribution, $S$, in the flow direction, $z$, is studied theoretically. With the assumptions of a slender liquid column and negligible gravitational and inertia effects, the problem is reduced to finding the axial velocity distribution at any cross section. The propagation of the liquid column, $h(z, t)$, and the tip location, $l(t)$, are then solved with the aid of the continuity equation. The following conclusions are drawn from the present study.

(1) When the half-wedge angle $\alpha$, the contact angle $\theta$, and the shear-stress distribution on the free surface $S$ are constant, analytic solutions exist. Otherwise, numerical simulation has to be applied.

(2) For a capillary flow in a wedge with constant $\alpha, \theta$, and $S$, both the analytic solutions and the numerical simulation predict that $l(t) \propto t^{3 / 5}$ for the constant-flow-rate stage and $l(t) \propto t^{1 / 2}$ for the constant-height flow stage.

(3) When $S$ is a function of the flow direction $z$, the above functional relationship between $l$ and $t$ becomes no longer valid; it varies as the liquid column propagates along the wedge.

\section{ACKNOWLEDGMENT}

Support from the National Center for Microgravity Research on Fluids and Combustion is greatly acknowledged.

${ }^{1}$ R. L. Columbus and H. J. Palmer, "Architectured fluid management of biological liquids," Clin. Chem. 33, 1531 (1987).

${ }^{2}$ J. R. Rollins, R. K. Grove, and D. E. Jaekle, Jr., "Twenty three years of surface tension propllant management system design, development, manu- facture, test, and operation," AIAA Pap. 85-1199 (1985).

${ }^{3} \mathrm{P}$. Concus and R. Finn, "On the behavior of a capillary surface in a wedge," Appl. Math. Sci. 63, 292 (1969).

${ }^{4} \mathrm{P}$. Concus and R. Finn, "On capillary free surfaces in the absence of gravity," Acta Math. 132, 177 (1974).

${ }^{5} \mathrm{P}$. Concus and R. Finn, "On capillary free surfaces in a gravitational field," Acta Math. 132, 207 (1974).

${ }^{6}$ A. de. Lazzer, D. Langbein, M. Dreyer, and J. Rath, "Mean curvature of liquid surfaces in containers of arbitrary cross-section," Microgravity Sci. Technol. 9, 208 (1996).

${ }^{7}$ G. Mason and N. Morrow, "Capillary behavior of a perfectly wetting liquid in irregular triangular tubes," J. Colloid Interface Sci. 141, 262 (1991).

${ }^{8}$ P. S. Ayyaswamy, I. Catton, and D. K. Edwards, "Capillary flow in triangular grooves,” Trans. ASME: J. Appl. Mech. 41, 332 (1974).

${ }^{9} \mathrm{M}$. Dong and I. Chatzis, "The imbibition and flow of a wetting liquid along the corners of a square capillary tubes," J. Colloid Interface Sci. 172, 278 (1995)

${ }^{10}$ W. B. Kolb and R. L. Cerro, "The motion of long bubbles in tubes of square cross section," Phys. Fluids A 5, 1549 (1993).

${ }^{11}$ B. Legait, "Laminar flow of two phases through a capillary tube with variable square cross-section," J. Colloid Interface Sci. 96, 28 (1983).

${ }^{12}$ H. B. Ma, G. P. Peterson, and X. Lu, "The influence of vapor-liquid interactions on the liquid pressure drop in triangular microgrooves," Int. J. Heat Mass Transfer 37, 2211 (1994).

${ }^{13}$ G. P. Peterson and H. B. Ma, "Analysis of countercurrent liquid-vapor interactions and the effect on the liquid friction factor," Exp. Therm. Fluid Sci. 12, 13 (1996).

${ }^{14}$ G. P. Peterson and H. B. Ma, "Laminar friction factor in micro-scale ducts of irregular cross section," Microscale Thermophys. Eng. 1, 253 (1997).

${ }^{15}$ T. C. Ransohoff and C. J. Radke, "Laminar flow of a wetting liquid along corners of a predominantly gas-occupied noncircular pore," J. Colloid Interface Sci. 121, 392 (1988).

${ }^{16}$ L. A. Romero and F. G. Yost, "Flow in an open channel capillary," J. Fluid Mech. 322, 109 (1996).

${ }^{17}$ M. M. Weislogel, "Capillary flow in an interior corner," Ph.D. thesis, Northwestern University (1996).

${ }^{18} \mathrm{M}$. M. Weislogel and S. Lichter, "A spreading drop in an interior corner: theory and experiment," Microgravity Sci. Technol. 9, 175 (1996).

${ }^{19}$ M. M. Weislogel and S. Lichter, "Capillary flow in an interior corner," J. Fluid Mech. 373, 349 (1998).

${ }^{20} \mathrm{D}$. Langbein, "The shape and stability of liquid menisci at solid edges," J. Fluid Mech. 213, 251 (1990).

${ }^{21}$ H. H. Hu and D. D. Joseph, "Lubricated pipelining: Stability of coreannular flow, Part 2," J. Fluid Mech. 205, 359 (1989).

${ }^{22}$ G. P. Peterson and H. B. Ma, "Theoretical analysis of the maximum heat transport in triangular grooves: a study of idealized micro heat pipes," J. Heat Transfer 118, 731 (1996).

${ }^{23}$ G. P. Peterson and H. B. Ma, "Temperature variation and heat transfer in triangular grooves with an evaporating film," J. Thermophys. Heat Transfer 11, 90 (1997).

${ }^{24}$ I. Catton and G. R. Stroes, "A semi-analytical model to predict the capillary limit of heated inclined triangular capillary grooves," J. Heat Transfer 124, $162(2002)$ 